\title{
Spherical-cap droplets of a photo-responsive bent liquid crystal dimer
}

2 Jun Yoshioka ${ }^{1}$, Péter Salamon ${ }^{2}$, Daniel A. Peterson ${ }^{3}$, John M. D. Storey ${ }^{3}$, Corrie T. Imrie ${ }^{3}$, Antal

$4 \quad{ }^{1}$ RIKEN Center for Emergent Matter Science (CEMS), 2-1 Hirosawa, Wako, Saitama 351-0198, Japan.

$6 \quad{ }^{2}$ Institute for Solid State Physics and Optics, Wigner Research Centre for Physics, Hungarian Academy of Sciences (HAS), H-1525 Budapest, P.O.B.49, Hungary.

${ }^{3}$ Department of Chemistry, School of Natural and Computing Sciences, University of Aberdeen, Meston Building, Old Aberdeen AB24 3UE, UK.

${ }^{4}$ Chemical Physics Interdisciplinary Program, Liquid Crystal Institute, Kent State University, Kent, OH 44242-0001, USA.

Using a photo-responsive dimer exhibiting the transition between nematic $(\mathrm{N})$ and twist-bend nematic $\left(\mathrm{N}_{\mathrm{TB}}\right)$ phases, we prepared spherical cap-shaped droplets on solid substrates exposed to air. The internal director structures of these droplets vary depending on the phase and on the imposed boundary conditions. The structural switching between the $\mathrm{N}$ and $\mathrm{N}_{\mathrm{TB}}$ phases was successfully performed either by temperature control or by UV light-irradiation. The $\mathrm{N}$ phase is characterized by an extremely small bend elastic constant $K_{3}$, and surprisingly, we found that the droplet-air interface induces a planar alignment, in contrast to that seen for typical calamitic liquid crystals. As a consequence, the director configuration was stabilized in a structure substantially different from that normally found in conventional nematic liquid crystalline droplets. In the twist-bend nematic droplets characteristic structures with macroscopic length scales were formed, and they were well controlled by the droplet size. These results indicated 
1 that a continuum theory is effective in describing the stabilization mechanism of the macroscopic

2 structure even in the twist-bend nematic liquid crystal droplets exhibiting director modulations

3 on a scale of several molecular lengths.

4

5 PACS numbers: $61.30 . P q$ (Microconfined liquid crystals: droplets, cylinders, randomly confined

6 liquid crystals, polymer dispersed liquid crystals, and porous systems), 64.70.Md (Transitions in

7 liquid crystals)

9 I. INTRODUCTION

10 In liquid crystals (LC) bounded by curved interfaces, geometrical frustrations play an

11 important role in the formation of various topological states. For example, it is well-known that

12 nematic $(\mathrm{N})$ materials show unique structures in a spherical droplet or shell geometry bounded

13 by an interface with a certain anchoring condition, because a geometrical frustration occurs in

14 such a spherically confined state [1-12 Candau-Liang2]. Spherical-cap geometries, such as a N

15 LC droplet placed on a flat solid substrate, are a little more complicated, because the N LC is

16 now surrounded by two different boundary conditions [13 Gupta].

17 In addition to the boundary conditions, elastic anisotropy also plays an important role. For 18 example, spherical droplets with strong planar anchoring often exhibit the so-called 'bipolar' 19 state, in which the $\mathrm{N}$ director field has two surface point defects. On the other hand, it can be 20 transferred into the so-called 'axial' state with a central line defect, depending on the ratio of 21 splay and bend elastic constants [3 Drzaic]. In addition, weak twist elasticity (low twist elastic

22 constant) allows the system to be twist-preferred. Helix formation through the spontaneous twist

23 deformation has been well-known even in N LCs of achiral molecules [5-8 KUJeong, Yang, 
2 Cholesteric $(\mathrm{Ch})$, smectic $(\mathrm{Sm})$, or columnar (Col) LC systems with curved boundaries are 3 more complicated [14-31 Yoshioka1-Oswald], because the director structures are dominated not 4 only by the two factors described already but also by others, such as helix formation due to the 5 chirality or the compression elasticity due to the translational ordering with molecular length 6 scales. The key point to be noticed here is the role played by frustration among these factors. 7 For instance, spherical $\mathrm{Ch}$ droplets show multi-dimensional twists even without the aid of 8 surface anchoring [14-16Yoshioka1, Ito, Poy]. In other words, the use of strong anchoring may 9 cause additional frustrations by which various complex topological states are realized in such 10 droplets [1, 17-24 Candau, Xu-Yoshioka2]. In this sense, LC materials showing the recently11 found twist-bend nematic $\left(\mathrm{N}_{\mathrm{TB}}\right)$ phase are attractive systems to study complex frustrations 12 induced by the curved surface. The $\mathrm{N}_{\mathrm{TB}}$ phase is characterised by spontaneous twist and bend 13 deformations due to the nanoscale heliconical structure [32-49 Dozov-Meyer4], which 14 theoretically can be treated in the framework of a course-grained description [32, 34, 38, 39, 41, 15 42, 45, 46 Dozov, Yun, Meyer2, Shamid, Greco, Challa, Parsouzi, Meyer3].

16 This paper focuses on the internal structures of spherical-cap droplets formed by a 17 photoresponsive bent-dimer that exhibits the $\mathrm{N}-\mathrm{N}_{\mathrm{TB}}$ phase transition. In general, the $\mathrm{N}$ phases of 18 such bent-dimers are characterized by extremely small bend elastic constants [33-36 Borshch, 19 Yun, Sebastián, Adlem]. In addition, the LC-air interface of the present dimer system shows 20 planar anchoring, in contrast to the majority of conventional calamitic $\mathrm{N}$ systems, for which the 21 alignment is normal to the air interface. Thus, the frustration between elastic anisotropy and the 22 boundary conditions is quite unique even in the $\mathrm{N}$ phase of this dimer. In contrast to the previous 23 studies dealing with the smectic-like bâtonnets in the coexisting state between the N-N $\mathrm{N}_{\text {TB }}$ phase 
transition [48,49 Krishnamurthy, Meyer4], this paper pays particular attention to the sphericalcap geometry in both phases with two different interfacial conditions, one at the flat substrate and the other air. An additional feature of note in the present study is the photo-responsiveness due to the azobenzene moiety, which allows a photo-induced $\mathrm{N}_{\mathrm{TB}}-\mathrm{N}$ transition to be driven by ultraviolet (UV) light irradiation [47, Paterson]. Here we also demonstrate the dynamic structure variation during the transition between the $\mathrm{N}$ and $\mathrm{N}_{\mathrm{TB}}$ phases.

\section{EXPERIMENTAL}

\section{A. Sample preparation}

The bent-dimer we used, $\mathrm{CB} 6 \mathrm{OABOBu}$ [47, Paterson], shows a direct transition from $\mathrm{N}$ to $\mathrm{N}_{\text {TB }}$ phase on cooling, and vice versa on heating. Since the $\mathrm{N}$ phase of this material appears in a relatively high temperature region $\left(\mathrm{Cr} 103^{\circ} \mathrm{C} \mathrm{N} \mathrm{N}_{\mathrm{TB}} 105^{\circ} \mathrm{C} \mathrm{N} 157^{\circ} \mathrm{C}\right.$ Iso), we mixed the dimer with a conventional room temperature N LC (5CB, Tokyo Chemical Industry Co., Ltd.) to lower the transition temperature between $\mathrm{N}$ and $\mathrm{N}_{\text {Тв }}$ [40, 44 Balachandran, Tuchband]. A sample mixture of $70 \% \mathrm{CB} 6 \mathrm{OABOBu}$ by weight fraction yielded the phase sequence, $\mathrm{Cr}-70^{\circ} \mathrm{C}-\mathrm{N} \mathrm{TB}-73^{\circ} \mathrm{C}-\mathrm{N}$ $120^{\circ} \mathrm{C}$-Isotropic (Iso), which was used in the present study. Critically, the photo-induced transition is possible even in the $70-30 \%$ mixture.

As for the droplet fabrication, 1-2 microliters chloroform solution with $0.1 \mathrm{wt} \%$ sample mixture content was cast on a glass substrate, followed by subsequent drying at above $100^{\circ} \mathrm{C}$ for about 1 hour to completely remove the chloroform. Due to this evaporation process, micronsized spherical-cap droplets are spontaneously formed on the substrate. Since the topological structure of the spherical-cap droplets depends on the anchoring of the substrate surface, we prepared three types of substrates: i) bare glass (washed by sonication in an alkaline detergent 
1 and rinsed several times with pure water); ii) unidirectionally rubbed glass coated with polyvinyl

2 alcohol (rubbed-PVA, Aldrich), and iii) non-rubbed glass coated with polyimide (JALS204, JSR

3 Corp.). In typical calamitic liquid crystals, strong planar anchoring is induced at the PVA-coated

4 substrate, whereas homeotropic is obtained at the substrate coated with JALS204 (Homeotropic 5 PI).

6

\section{B. Polarizing microscopy and optical simulation}

8 The textures of the droplets were observed by polarizing microscopy (POM) using a 9 commercial microscope (BH2, Olympus) and a digital camera (EOS Kiss X50, Canon). The

10 observations were performed not only with crossed polarizers but also with an additional tint 11 plate (retardation $\lambda=530 \mathrm{~nm}$ ) for sensitive colour observation. Temperature control was achieved

12 using a custom-made hot-stage, while the temperature inside the droplets was estimated to be

13 lower than that in the stage because the present system was exposed to the air. In fact, the N-N $\mathrm{N}_{\mathrm{TB}}$

14 transition occurred when the temperature in the stage was 15-25 degrees higher than the 15 transition temperature.

16 Based on the POM images we simulated the internal director fields using the Jones matrix

17 method [50 Jones]. Spectral transmittances were calculated for 22 different wavelengths, and 18 then converted into RGB values using the colour-matching function. The incident light was 19 assumed to be a flat white light having an equal intensity over the calculated wavelength range.

20 The ordinary refractive index $n_{o}$ was assumed to be 1.5 according to Ref. [51 Vaz]. A 21 commercial spectroscope (USB2000+VIS-NIR, Ocean Optics) was used to obtain the 22 extraordinary refractive index $n_{e}[52 \mathrm{Kleman}]$ and the birefringence $\Delta n=n_{e}-n_{o}$. A mercury lamp 23 (U-LH100HG equipped on BX53W, Olympus) was used for the in-situ observation of the photo- 
1 induced phase transition under UV irradiation.

\section{Confocal reflection microscopy (CRM)}

4 A confocal reflection microscope (TCS SP8, Leica) was used to estimate the height of the 5 spherical-cap droplets. We used a dry objective lens with a numerical aperture of 0.7 , which

6 corresponds to a focal depth of about $2.4 \mu \mathrm{m}$ for the optical wavelength of $500 \mathrm{~nm}$. The

7 excitation and observation were made on the LC-air interface of the spherical-cap droplets.

8 CRM analyses were carried out to measure the heights and contact angles of these droplets.

9 Figure 1(a)-(d) is an example of the CRM observations, showing the clear reflections at the

10 substrate-air and LC-air interfaces, by which we could confirm the spherical-cap shape of the

11 droplets. The droplet height $h$ estimated from the cross-sectional CRM profile was plotted as a

12 function of the radius $R$. As shown in Fig. 1(e), $h$ is almost proportional to $R$, irrespective of 13 surface treatment and observed phases. Thus, by assuming linearity between them, we can 14 describe the contact angle $\theta_{c}$ as,

$$
\theta_{c}=2 \arctan \left(\frac{h}{R}\right)
$$

$16 \theta_{c}$ values deduced from the CRM data with the $\theta / 2$ method [53 Yang2] are summarized in Table

17 I. The contact angle depends strongly on the surface treatment of the glass: the angle was found 18 to be largest for the homeotropic PI, and smallest for the rubbed PVA.

\section{TEXTURES}

\section{A. Temperature and surface treatment dependence}

22 Figure 2 shows POM micrographs as viewed from the top of the spherical-cap droplets in $\mathrm{N}$ 
1 and $\mathrm{N}_{\mathrm{TB}}$ phases on the three types of substrates. The $\mathrm{N}$ droplets on the bare glass have a

2 'bipolar'-type texture (Fig. 2(a) and (a')) with two defect points. Extinction occurs for every $90^{\circ}$

3 rotation of the sample indicating a uniform director field for each droplet, although its azimuthal

4 director angle changes randomly by every cycle of heating and cooling between Iso and $\mathrm{N}$.

5 Similar concentric colours are observed for the $\mathrm{N}$ droplets on the rubbed PVA (Fig. 2(b) and

$\left.6\left(b^{\prime}\right)\right)$, although the defect points are not so clear as those formed in the N droplets on bare glass.

7 Again, extinction is confirmed for every $90^{\circ}$ rotation of the sample, implying a unidirectional

8 alignment. However, the director in this case is always aligned to the rubbing direction indicated

9 by green arrows. On homeotropic PI substrates a spiraling extinction cross appears in the $\mathrm{N}$

10 phase (Fig. 2(c) and $\left(\mathrm{c}^{\prime}\right)$ ). Note that the spiraling direction is not given uniquely, i.e. the

11 handedness may change after melting. This fact confirms that the whole system is achiral, and

12 the swirling pattern is not due to an intrinsic chirality.

13 In all cases, the textures alter on cooling through the $\mathrm{N}$ to $\mathrm{N}_{\mathrm{TB}}$ phase transition. For both bare 14 glass (Fig. 2(d) and (d')) and rubbed PVA (Fig. 2(e) and (e')), the stripe domains are decorated 15 with a shorter periodicity stripe pattern. The direction of the boundaries of these stripe domains 16 corresponds to the $\mathrm{N}$ director, i.e., in the case of the rubbed PVA, it is parallel to the rubbing 17 direction.

18 For the homeotropic PI, a simple smectic A ( SmA) type extinction cross is observed for 19 relatively small droplets (Fig. 2(f)), which becomes more complicated in larger droplets, 20 showing two distinguishable inner and outer regions (Fig. 2(f')). A circular domain with a 21 swirling cross appears in the inner region resembling the $\mathrm{N}$ droplet on homeotropic PI. The outer 22 region shows a non-twisted extinction cross with SmA-type fan-shaped domains. Since the 23 nanoscale-pitch of the heliconical structure can be considered to be pseudo-layers, most of the 
1 textural features can be explained by analogy to the SmA phase [49 Meyer4].

\section{B. Photo-induced transition}

4 As reported earlier, UV illumination of $\mathrm{CB} 6 \mathrm{OABOBu}$ induces a reversible transition from $\mathrm{N}_{\mathrm{TB}}$

5 to $\mathrm{N}$ [47 Paterson]. Textural changes could be induced also in our mixtures using $0.6 \mathrm{~mW}$

6 intensity light. Specifically, we found that the stripe domains formed in the $\mathrm{N}_{\mathrm{TB}}$ droplets on bare

7 glass and rubbed PVA could be erased using UV (see Fig. 3(a) and (b)) and re-appeared after the

8 UV was turned off, (Fig. 3(a') and (b') and Movies S1(a)-S1(d) in Supplementary Material

9 (SM) 1). This phenomenon can be understood as a result of the reversible photo-induced

10 transitions between $\mathrm{N}_{\text {TB }}$ and $\mathrm{N}$.

11 The UV-induced textural changes for larger droplets on non-rubbed PI is more complex. First

12 the outer SmA-type domains melt into a smooth N-type texture, while the inner region remains

13 unchanged (see Fig. 3(c) and Movie S1(e) in SM 1). Further UV irradiation completes the

14 transition process with the transformation in the inner region, so the entire droplet became almost

15 identical to the $\mathrm{N}$ droplet with a spiraling cross. When the UV irradiation is switched off, 16 spiraling fringes appear first, as shown in Fig. 3(c') and Movie S1(f) in SM1. This fringe state

17 persists for a few seconds, and then the inner circular region re-appears to restore the initial 18 focal-conic state of the $\mathrm{N}_{\text {тв }}$ droplet.

\section{STRUCTURAL PROPERTIES}

\section{A. $\mathbf{N}$ droplet structure}

22 1. On bare glass

23 As described earlier, on bare glass the $\mathrm{N}$ droplets show 'bipolar'-type textures (Fig. 2(a) and 
$\left.1 \quad\left(a^{\prime}\right)\right)$ often observed in spherical $\mathrm{N}$ droplets surrounded by a surface with planar anchoring [1-3

2 Candau, Volovic, Drzaic]. However, in the present case, two apparent defects are not located at

3 the edge, but slightly inside the droplet. By rotating the sample by $45^{\circ}$, two-fold brushes

4 corresponding to $s^{=+1 / 2}$ defects are observed (see red arrow in Fig. 2(a')), unlike the usual

5 'bipolar' state with the defect $s=+1$. In addition to the crossed-polarizer condition, we conducted

6 POM observation under the birefringence-sensitive state using a tint plate to visualize even slight

7 deformations in the director field. As shown in Fig. 4(a), very slight colour deviation from

8 purple to blue or yellow (the blue and orange ellipses) can be seen in the edge regions at $\pm 45^{\circ}$

9 with respect to the polariser direction. Thus, this observation confirms the existence of a slight

10 deformation of the director field due to the planar anchoring and the curvature of the air-LC

11 interface. Since blue and yellow correspond to additive and reductive birefringence colours

12 according to the Michel-Levi chart, respectively, the director configuration is identified as

13 indicated by the yellow and blue ellipses in Fig. 4(a). It should be noted here that this anchoring

14 condition is the opposite from that in a conventional calamitic $\mathrm{LC}$, such as $5 \mathrm{CB}$. We return to

15 this unusual observation at the end of section IV-A3.

16 Based on these observations, it is clear that the circular $\mathrm{N}$ director field is tangential along the

17 droplet edge. Hence, two $s=+1 / 2$ line defects appear slightly inside, instead of the $s=+1$ point

18 defects at the edge as drawn in Figs. $4\left(\mathrm{~b}^{\prime \prime}\right)$ and $\left(\mathrm{c}^{\prime \prime}\right)$. This indicates that the bend deformation is

19 more favorable than the splay deformation around the edge and the defects. This is because in

20 bent-dimers exhibiting the $\mathrm{N}_{\text {Tв }}$ phase the bend elastic constant is significantly smaller than the

21 splay and twist elastic constants [33-36 Borshch-Adlem]. In the present case, the bend

22 deformation along the round edge and the $s=+1 / 2$ defects are preferred over the more costly

23 vertical splay at the edge and the splay around the $s=+1$ defects. In addition, in the present 
1 system, the azimuthal anchoring on the bare glass substrate should be sufficiently weak for the

2 director to follow the circular edge alignment. Although these two line defects repel each other,

3 they are also pushed inside by the edge. Thus, the defect positions are determined by the balance

4 of these repulsive forces. Note that such a $s=+1 / 2$ structure is topologically impossible in

5 spherical droplets and to the best of our knowledge, there is no report of such a structure in a

6 stationary state even in the spherical-cap geometry.

7 According to the above considerations, the director field was approximated by the following 8 trial functions,

$$
\begin{aligned}
& n_{r}=\cos \left(\frac{\alpha z r \cos \left(\phi-\phi_{0}\right)}{h R}\right) \cos \left(\phi-\phi_{0}-\phi_{n}\right) \\
& n_{\varphi}=-\cos \left(\frac{\alpha z r \cos \left(\phi-\phi_{0}\right)}{h R}\right) \sin \left(\phi-\phi_{0}-\phi_{n}\right), \\
& n_{z}=\sin \left(\frac{\alpha z r \cos \left(\phi-\phi_{0}\right)}{h R}\right)
\end{aligned}
$$

where

$$
\begin{aligned}
& \phi_{n}= \begin{cases}-\phi_{p} / 2 & \text { for }\left(0<\phi-\phi_{0}<\pi\right) \\
\phi_{p} / 2 & \text { for }\left(-\pi<\phi-\phi_{0}<0\right)\end{cases} \\
& \phi_{p}= \begin{cases}-\arctan \zeta_{+} & \text {for }\left(\pi / 2<\phi-\phi_{0}<\pi\right) \\
\arctan \zeta_{+} & \text {for }\left(0<\phi-\phi_{0}<\pi / 2\right) \\
\arctan \zeta_{-} & \text {for }\left(-\pi / 2<\phi-\phi_{0}<0\right) \\
-\arctan \zeta_{-} & \text {for }\left(-\pi<\phi-\phi_{0}<-\pi / 2\right)\end{cases} \\
& \zeta_{ \pm}=\frac{r \cos \left(\phi-\phi_{0}\right) \sin \phi_{d}}{r \sin \left(\phi-\phi_{0}\right) \sin \phi_{d} \mp R_{d} \sin \phi_{d}} \\
& \phi_{d}= \begin{cases}\arctan \xi & \text { for }\left(0<\phi-\phi_{0}<\pi\right) \\
-\arctan \xi & \text { for }\left(-\pi<\phi-\phi_{0}<0\right)\end{cases} \\
& \xi=\frac{2 r R_{d} \sin \left(\phi-\phi_{0}\right)}{r^{2}-R_{d}^{2}}
\end{aligned}
$$

12 For this model structure the range of the parameters should be set as, 


$$
\begin{array}{cc}
0<\phi_{p}, \phi_{d}<\pi & \text { for }\left(0<\phi-\phi_{0}<\pi\right) \\
-\pi<\phi_{p}, \phi_{d}<0 & \text { for }\left(-\pi<\phi-\phi_{0}<0\right)
\end{array} .
$$

2 Here, the cylindrical coordinate system is set at the substrate plane in such a way that the $z$-axis

3 is perpendicular to the substrate. $\phi_{0}$ and $R_{d}$ correspond to the azimuthal angle of the direction

4 connecting two $s=+1 / 2$ defects and the midway distance between them, respectively. Finally, $\alpha$

5 is a dimensionless parameter. To validate this structural model, we simulated the spatial

6 distribution of the light transmission intensity between crossed polarizers using the Jones matrix

7 method. The simulated POMs (Fig. 4(a')) are compared with the experimental observations

8 (Fig. 4(a)). Apparently, even detailed features of the birefringent colours are reproduced,

9 particularly those around the defect points, validating our structural model. We would like to

10 emphasize that this $s=+1 / 2$ defect structure is unique to the present system characterized by the

11 small bend elastic constant, strong planar anchoring at the LC-air interface, and weak planar

12 anchoring at the LC-substrate interface.

\section{Rubbed-PVA}

15 Unlike on the bare glass substrate, the director at the surface of the rubbed PVA is strongly 16 anchored and aligned almost unidirectionally along the rubbing direction (Fig. 2(b) and (b')).

17 Figure 5(a) shows the POM textures captured with the tint plate. Again, blue and orange colours 18 are observed at the edge regions, which means the director field is deformed along the round 19 edge. Since uniform alignment is achieved only in the vicinity of the LC-substrate interface, the 20 director is continuously deformed towards the LC-air interface with strong planar anchoring 21 which forces the director to be aligned along the curved LC-air interface. The small contact 22 angle (high wettability, see Table I) with respect to the substrate may reduce the vertical splay at 23 the edge, therefore the system can accept and maintain the 'bipolar' state more likely than on 
1 bare glasses. Based on these observations, the director field is assumed to be as shown in the

2 cartoons in Figs. 5(b)-(b') and (c)-(c's). The only concern with this model is that no clear defect

3 is observed in POM, although such a confined state with a closed boundary topologically

4 requires defects. However, it is possible because of the small contact angle, that such surface

5 point defects cause only small deformations and are hidden at the edge region. It is clear that on

6 the PVA-coated substrate, the droplets have the splay deformation at the surface, in contrast to

7 those on the bare substrate.

8 Such a director field can be approximated as,

$$
\begin{aligned}
& n_{r}=\cos \left(\frac{\alpha z r \cos \left(\phi-\phi_{0}\right)}{h R}\right) \cos \left(\phi-\phi_{0}+\frac{\beta z r^{2} \sin 2\left(\phi-\phi_{0}\right)}{2 h R^{2}}\right) \\
& n_{\phi}=-\cos \left(\frac{\alpha z r \cos \left(\phi-\phi_{0}\right)}{h R}\right) \sin \left(\phi-\phi_{0}+\frac{\beta z r^{2} \sin 2\left(\phi-\phi_{0}\right)}{2 h R^{2}}\right) . \\
& n_{z}=\sin \left(\frac{\alpha z r \cos \left(\phi-\phi_{0}\right)}{h R}\right)
\end{aligned}
$$

10 The coordinates and the parameters are defined similarly to Eq. (2), except for the non-

11 dimensional parameter $\beta$. The director field given by Eq. (3) does not have defects, and the

12 simulated POM images perfectly reproduce the experimental POMs, $c f$. Figs. 5(a) and (a'). In

13 this case, the strong unidirectional anchoring of the rubbed PVA surface retains the splay

14 deformations at the edge whereas in the case of bare glass with weak anchoring, the director

15 escapes from the splay into the bend around the $s=+1 / 2$ line defects.

\section{3. Homeotropic PI}

18 In contrast to the two previous cases in which both the LC-air and the LC-substrate interfaces 19 have planar anchoring, here the LC-substrate interface has homeotropic anchoring, while the LC20 air interface maintains planar anchoring. In such a case the director field is generally considered 
1 to become conically symmetric (Fig. S1(a) in SM 2), and the resulting texture has the so-called

2 'Maltese-cross' pattern. However, the actual POM texture shows a spiraling pattern (Figs. 2(c)

3 and $\left.\left(\mathrm{c}^{\prime}\right)\right)$, indicating a twisted director field, as described by the trial functions,

4

$$
\begin{aligned}
& n_{r}=\sin \left(\frac{\pi z}{2 h}\right) \cos \left(\frac{\beta(R-r)}{R}\right) \\
& n_{\phi}=-\sin \left(\frac{\pi z}{2 h}\right) \sin \left(\frac{\beta(R-r)}{R}\right) . \\
& n_{z}=-\cos \left(\frac{\pi z}{2 h}\right)
\end{aligned}
$$

5 Again, the coordinates and the parameters are defined similarly to the cases for Eqs. (2) and (3).

6 It should be noted that $\beta$ is a parameter for the twist deformation along the radial direction, and $7 \quad \beta=0$ gives a conical director structure with a splay deformation as illustrated in Fig. S1 in SM 2. However, when $\beta$ takes non-zero values, the twist deformation is induced along the radial

9 direction, by which the bend deformation is partially replaced by splay. Finally, $\beta= \pm \pi / 2$ gives a

10 ring-like director field with the bend around the central axis of the droplet, while the splay 11 deformation is maintained at the edge region as shown in Figs. 6(b') and (c'). The simulated 12 POM image with $\beta=\pi / 2$ in Fig. 6(a') agrees well with the real image shown in Fig. 6(a). This 13 structure is also energetically favored because the bend elastic constant is the smallest [33-36 14 Borshch-Adlem]. The escape from the splay to a bend and twist dominant structure leads to the 15 spiraling structure.

16 In contrast to the present case, conventional NLCs, such as 5CB, show line defects only with 17 planar anchoring at the substrate and homeotropic anchoring at the LC-air interface [13 Gupta]. 18 The difference between the anchoring properties at the LC-air interface of conventional NLCs 19 and the present dimer, presumably reflects differences in their respective structural. Thus the 20 unusual planar anchoring at the air interface for the dimer is probably due to the placement of the 
1 flexible hydrocarbon chains in the center of the molecule instead of at the end as in conventional

2 LCs. For entropy reasons the alkyl chains prefer to be in contact with air, inducing homeotropic 3 alignment in 5CB and planar for $\mathrm{CB} 6 \mathrm{OABOBu}$.

4

\section{B. Structures of $\mathrm{N}_{\mathrm{TB}}$ droplets}

6 As described earlier, the $\mathrm{N}_{\mathrm{TB}}$ droplets on the bare glass and the rubbed PVA substrates show

7 stripes decorated with zigzag undulations. Such stripes and undulations are often observed in

8 smectic A (SmA) shells with finite thickness [10, 11, 25, 26 Liang1, Lopez-Leon, Manyuhina,

9 Lee] and for the $\mathrm{N}_{\text {TB }}$ phase in sandwich cells with planar anchoring conditions [27, 35, 40 Yun,

10 Challa, Paterson]. In smectics, the requirement for the constant layer spacing prohibits the bend

11 deformation [54 de Gennes]. Therefore, frustration occurs in the spherical shells with planar

12 anchoring, often resulting in the periodic buckling of the layered structure $[10,11,25,26$ Liang1,

13 Lopez-Leon, Manyuhina, Lee]. Similarly, the nanoscale heliconical arrangement of the $\mathrm{N}_{\mathrm{TB}}$

14 phase is considered as a pseudo-layer structure, so that the constraints imposed by the curved

15 surface with planar anchoring trigger the buckling instability of the pseudo-layers [34, 42, 47

16 Yun, Challa, Paterson]. Since the pseudo-layers may shrink on cooling, this can also trigger the

17 periodic buckling (Fig. 7(a)).

18 Previous studies of SmA and $\mathrm{N}_{\mathrm{TB}}$ LCs showed that the stripe period increases with sample

19 thickness [REF]. In Fig. 7(b) we plot the stripe period $d$ and the droplet height $h$ as a function of

20 the droplet radius $R$. Both for the bare glass and the rubbed PVA, $d$ and $h$ are proportional to $R$

21 and are almost equal. This renforces the view that the formation of the stripe pattern in our $\mathrm{N}_{\mathrm{TB}}$

22 droplets may be described using a similar mechanism to that for the stripes formed in the SmA

23 shells or by the $\mathrm{N}_{\mathrm{TB}} \mathrm{LC}$ in sandwich cells. 
Figure 2(f) shows that smaller $\mathrm{N}_{\mathrm{TB}}$ droplets on homeotropic PI appear similar to circular domains of Sm LCs, emphasizing the pseudo-layer nature of the nanoscale heliconical structure of the $\mathrm{N}_{\text {TB }}$ phase. Larger $\mathrm{N}_{\mathrm{TB}}$ droplets however have different structures in the inner and outer regions as shown in Fig. 2(f'). This structure is different from the bunch structures of radiallyarranged focal-conics of Sm droplets reported previously [26-30 Lee, Naito, Meyer1, Gharbi, Beller]. The striped texture of the outer region can be understood as a consequence of the $\mathrm{N}_{\mathrm{TB}}$ pseudo-layer structure. The texture of the circular inner domain however is similar to that we found in the $\mathrm{N}$ phase (see Fig. 2(c')). The inner circular region seems to have a distinct size variation on the droplet radius $(R)$ and droplet height $(h)$ as shown in Fig. 7(c). Similar to the case of the planar anchoring, $h$ shows linear dependence on $R$ (purple dots) although with a steeper slope due to the larger contact angle (see Table I). However, $R_{c}$, being a few times smaller than the droplet height $h$ (red squares), shows a non-linear dependence on $R$. From the texture, we qualitatively model a plausible structure as sketched in Fig. S3(a) in SM 3, in which the droplet consists of three regions, Regions I-III. Region I is the outermost part containing stripes. Here, the pseudo-layers are considered to be bent and the helical axis of which is splayed to connect the planar alignment at the air interface and the homeotropic alignment at the substrate. The stripes are a result of the buckling of the pseudo layers due to the decrease of the pitch on cooling. Region II is the cylindrical part in the inner region, surrounded by Region I. Region II also has pseudo-layers but parallel to the substrate, and thus no deformation of the pseudo-layers to cost the deformation free energy. This region can avoid buckling by changing its radius to compensate for the pitch variation on cooling. Region III is the spherical-cap part on the top of Region II, whose height is estimated to be several hundreds of nanometers (for more details of this estimation, see SM 3). Since this region is a thin layer sandwiched between two 
1 different boundaries of homeotropic and planar alignment conditions, strong geometrical

2 frustration occurs. As a result, Region III melts into nematic ordering without the twist-bend

3 structure. Since Region II is optically isotropic and hence does not contribute to the birefringence

4 color, the texture of the inner part reflects only the features of Region III. Therefore, it looks like

5 a nematic droplet embedded in the center. Note that smaller droplets only consist of Region I.

\section{V. SUMMARY}

8 In this paper, we prepared spherical-cap droplets on glass substrates exposed to the air using 9 the photo-responsive bent-shaped dimer $\mathrm{CB} 6 \mathrm{OABOBu}$ which shows the $\mathrm{N}$ and $\mathrm{N}_{\mathrm{TB}}$ phases. In

10 the droplets, the switching of these two phases was successfully performed using a change in 11 temperature or by UV light irradiation. In addition, to control the structure inside the droplets, 12 we prepared three types of the substrates: bare, PVA-coated and homeotropic PI-coated glasses.

13 Structures in the $\mathrm{N}$ droplets are different from those seen for conventional calamitic nematic 14 droplets due to the anomalously low director bend elastic constant of bent dimers. POM 15 observations of the $\mathrm{N}$ droplets revealed that the anchoring condition at the air-LC interface is a 16 planar alignment in contrast to the homeotropic alignment typical for calamitic nematics, such as

17 5CB. On bare glass and PVA coated substrates, 'bipolar'-type structures are formed in the 18 droplets. However, the detailed structures were different for these two cases: on the bare glass 19 substrate, a pair of $+1 / 2$ line defects formed inside the droplet to avoid the splay and induce bend 20 deformation, while on the PVA-coated substrate, the defects are not formed but slight director 21 deformations were induced at the edge, owing to the strong planar anchoring and the low contact 22 angle. On the homeotropic PI, despite the absence of molecular chirality, a twisted director field 23 with a spiraling Maltese cross formed, as this structure minimized the costly splay deformations. 
In the $\mathrm{N}_{\mathrm{TB}}$ droplets, the structures were more complex. On bare and PVA-coated substrates, a stripe pattern with a period $d$ of several to several tens of micrometres was observed. This pattern formation may be explained by the periodic buckling of the pseudo-layers of the $\mathrm{N}_{\mathrm{TB}}$ phase due to geometrical frustration induced by the surface with planar anchoring, by analogy with spherical SmA shells or $\mathrm{N}_{\mathrm{TB}} \mathrm{LC}$ in sandwich cells. On homeotropic PI, a SmA-like fanshaped texture was observed for smaller droplets. For larger droplets, a circular domain of several micrometers with a N-like smooth texture at its centre appeared. We proposed a structural model that can qualitatively explain the formation of this nematic-like inner region.

\section{Acknowledgement}

The stays and research activities of J.Y. and F.A. in Hungary, and P.S. and A.B. in Japan are supported by the JSPS-HAS bilateral program. J.Y. was partially supported by JSPS KAKENHI Grant Number 15K17739. A.J. acknowledges financial support by NSF DMR: 1307674. Financial support from the grants NKFIH PD 121019 and FK 125134 are acknowledged.

\section{References}

[1 Candau] M. Candau, P. LeRoy, and F. Debeauvais, Mol. Cryst. Liq. Cryst. 23, 283 (1973).

[2 Volovik] G. E. Volovik and O. D. Lavrentovich, Sov. Phys. JETP 58, 1159 (1983).

[3 Drzaic] P. Drzaic, Mol. Cryst. Liq. Cryst. 154, 289 (1988).

[4 OndrisCrawford] R. OndrisCrawford et al., Appl. Phys. 69, 6380 (1991).

[5 KUJeong] K. -U. Jeong et al., Adv. Mater. 18, 3229 (2006).

[6 Yang] D. -K. Yang, K. -U. Jeong, and S. Z. D. Cheng, J. Phys. Chem. B 112, 1358 (2008).

[7 JJeong] J. Jeong et at., Proc. Natl. Acad. Sci. USA 111, 1742 (2014). 
1 [8 Ignés-Mullol] J. Ignés-Mullol, G. Poy, and P. Oswald, Phys. Rev. Lett. 117, 057801 (2016).

2 [9 Fernández -Nieves] A. Fernández-Nieves et al., Phys. Rev. Lett. 99, 157801 (2007).

3 [10 Liang1] H. -L. Liang, et al., Phys. Rev. Lett. 106, 247801 (2011).

4 [11 Lopez-Leon] T. Lopez-Leon et al., Phys. Rev. Lett. 106, 247802 (2011).

5 [12 Liang2] H. -L. Liang et al., Soft Matter 8, 5443 (2012).

$6 \quad$ [13 Gupta] V. K. Gupta and N. L. Abbot, Langmuir 15, 7213 (1999).

7 [14 Yoshioka1] J. Yoshioka, F. Ito, and Y. Tabe, Soft Matter 12, 2400 (2016).

8 [15 Ito] F. Ito, J. Yoshioka, and Y. Tabe, J. Phys. Soc. Jpn. 85, 114601 (2016).

9 [16 Poy] G. Poy, F. Bunel, and P. Oswald, Phys. Rev. E 96, 012705 (2017).

10 [17 Xu] F. Xu and P. P. Crooker, Phys. Rev. E 56, 6853 (1997).

11 [18 Seč1] D. Seč, et al., Soft Matter 8, 11982 (2012).

12 [19 Seč2] D. Seč, S. Čoper, and S. Žumer, Nat. Commun. 5, 3057 (2014).

13 [20 Orlova] T. Orlova, et al., Nat. Commun. 6, 7603 (2015).

14 [21 Posnjak1] G. Posnjak, S. Čoper, and I. Muševič, Sci. Rep. 6, 26361 (2016).

15 [22 Posnjak2] G. Posnjak, S. Čoper, and I. Muševič, Nat. Commun. 8, 14594 (2017).

16 [23 Krakhalev] M. K. Krakhalev et al., Sci. Rep. 7, 14582 (2017).

17 [24 Yoshioka2] J. Yoshioka and F. Araoka, Nat. Commun. 9, 432 (2018).

18 [25 Manyuhina] O. V. Manyuhina and M. J. Bowick, J. Non-Linear Mech. 75, 87 (2015).

19 [26 Lee] G. Lee et al., Part. Part. Syst. Charact. 30, 1-7 (2013).

20 [27 Naito] H. Naito, M. Okuda, and O -Y. Zhong-can, Phys. Rev. E 52, 2095 (1995).

21 [28 Meyer1] C. Meyer et al., Materials 2, 499 (2009).

22 [29 Gharbi] M. A. Gharbi et al., Langmuir 3111135 (2015).

23 [30 Beller] D. A. Beller et al., Phys. Rev. X 3, 041026 (2013). 
1 [31 Oswald] P. Oswald and P. Pieranski, Smectic and Columnar Liquid Crystals, (CRC Press,

2 Boca Raton, 2006).

3 [32 Dozov] I. Dozov, Europhys. Lett. 56, 247 (2001).

$4 \quad$ [33 Borshch] V. Borshch et al., Nat. Commun. 4, 2635 (2013).

5 [34 Yun] C. -J. Yun et al., Appl. Phys. Lett. 106, 173102 (2015).

6 [35 Sebastián] N. Sebastián et al., Phys. Chem. Chem. Phys. 18, 19299 (2016).

7 [36 Adlem] K. Adlem et al., Phys. Rev. E 88, 22503 (2013).

$8 \quad$ [37 Chen] D. Chen et al., Proc. Natl. Acad. Sci. USA 110, 15931 (2013).

9 [38 Meyer2] C. Meyer, G. R. Luckhurst, and I. Dozov, Phys. Rev. Lett. 111, 067801 (2013).

10 [39 Shamid] S. M. Shamid, S. Dhakal, and J. V. Selinger, Phys. Rev. E 87, 052503 (2013).

11 [40 Balachandran] R. Balachandran et al., J. Mater. Chem. C 2, 8179 (2014).

12 [41 Greco] C. Greco, G. R. Luckhurst, and A. Ferrarini, Soft Matter 10, 9318 (2014).

13 [42 Challa] P. K. Challa et al., Phys. Rev. E 89, 060501 (2014).

14 [43 Gorecka] E. Gorecka et al., Angew, Chem. 54, 10155 (2015).

15 [44 Tuchband] M. R. Tuchband et al., arXiv:1511.07523 (2015).

16 [45 Parsouzi] Z. Parsouzi et al., Phys. Rev. X 6, 021041 (2016).

17 [46 Meyer3] C. Meyer and I. Dozov, Soft Matter 12, 574 (2016).

18 [47 Paterson] D. A. Paterson et al., J. Am. Chem. Soc. 138, 5283 (2016).

19 [48 Krishnamurthy] K. S. Krishnamurthy et al., Soft Matter 12, 4967 (2016).

20 [49 Meyer4] C. Meyer et al., Liq. Cryst. 44, 232 (2017).

21 [50 Jones] R. C. Jones, J. Opt. Soc. Am. 31, 488-493 (1941).

22 [51 Vaz] N. A. Vaz and G. P. Montgomery, J. Appl. Phys. 62, 3161-3172 (1987).

23 [52 Kleman] M. Kleman and O. D. Lavrentovich, Soft Matter Physics An Introduction 
1 (Springer, New York, 2003).

2 [53 Yang2] M. -W. Yang and S. -Y. Lin, Coll. Surf. A Physicochem. Eng. Aspects 220, 199 3 (2003).

4 [54 de Gennes] P. G. de Gennes and J. Prost, The Phisics of Liquid Crystals second edition, 5 (Clarendon Press Oxford, New York, 1993).

Table and Figure captions

9 TABLE I. Summary of the shape-dependent parameters, height-radius ratio $h / R$, contact angle $10 \theta_{\mathrm{c}}$, and pitch-radius ratio of the stripe domains $d / R$, of the $\mathrm{N}$ and $\mathrm{N}_{\mathrm{TB}}$ droplets.

12 FIG. 1. (a-d) Example of CRM images of the spherical-cap $\mathrm{N}_{\mathrm{TB}}$ droplet placed on the bare 13 glass substrate $\left(T \sim 60^{\circ} \mathrm{C}\right)$. Scale bars, $10 \mu \mathrm{m}$. (a-c) Horizontal cross-sectional images taken for 14 three different planes as described in (d). (d) Vertical cross-sectional image sliced at the 15 centre, constructed from the horizontal cross-sectional images obtained by vertical scanning

16 CFM. (e) The droplet height $h$ plotted as a function of the droplet radius $R$. Both $h$ and $R$ are

17 estimated from the vertical cross-section sliced at the centre, as shown in (e). Closed and 18 open symbols are for $\mathrm{N}$ and $\mathrm{N}_{\mathrm{TB}}$ droplets, respectively. Solid and dashed lines represent 19 linear-fitting to them. Rectangles, triangles, and circles denote the homeotropic PI, the bare 20 glass, and the rubbed PVA substrates, respectively. The temperature was $\sim 90^{\circ} \mathrm{C}$ for $\mathrm{N}$ and $21 \sim 60^{\circ} \mathrm{C}$ for $\mathrm{N}_{\mathrm{TB}}$ droplets. See also the summary of the slope values $h / R$, from which the 22 contact angle $\theta_{c}$ can be deduced. 
1 FIG. 2. Polarization micrographs of spherical-cap droplets in (a-c, $\left.\mathrm{a}^{\prime}-\mathrm{c}^{\prime}\right) \mathrm{N}\left(T \sim 90^{\circ} \mathrm{C}\right)$ and $(\mathrm{d}-\mathrm{f}$,

$\left.2 \mathrm{~d}^{\prime}-\mathrm{f}^{\prime}\right) \mathrm{N}_{\mathrm{Tв}}\left(T \sim 60^{\circ} \mathrm{C}\right)$ phases, placed on three different substrates, (a, $\left.\mathrm{a}^{\prime}, \mathrm{d}, \mathrm{d}^{\prime}\right)$ bare glass, $\left(\mathrm{b}, \mathrm{b}^{\prime}\right.$,

3 e, $\left.e^{\prime}\right)$ rubbed PVA, and $\left(\mathrm{c}, \mathrm{c}^{\prime}, \mathrm{f}, \mathrm{f}^{\prime}\right)$ homeotropic PI. Scale bar, $10 \mu \mathrm{m}$. The green arrows in the

4 rubbed PVA results represent the rubbing direction. Each image set of (a, $\left.a^{\prime}\right),\left(b, b^{\prime}\right),\left(d, d^{\prime}\right)$,

5 and $\left(\mathrm{e}, \mathrm{e}^{\prime}\right)$ was taken for the same droplet but with two different rotation angles, $(\mathrm{a}, \mathrm{b}, \mathrm{d}, \mathrm{e}) 0^{\circ}$

6 (extinction) and $\left(a^{\prime}, b^{\prime}, d^{\prime}, e^{\prime}\right) 45^{\circ}$ (bright state). The $\mathrm{N}_{\text {TB }}$ states $\left(\mathrm{d}, \mathrm{d}^{\prime}, \mathrm{e}, \mathrm{e}^{\prime}, \mathrm{f}, \mathrm{f}^{\prime}\right)$ are obtained

7 by cooling from $\left(a, a^{\prime}, b, b^{\prime}, c, c^{\prime}\right)$, respectively. Note that the droplets $(c, f)$ and $\left(c^{\prime}, f^{\prime}\right)$ are

8 different, specifically, $(\mathrm{c}, \mathrm{f})$ small, and $\left(\mathrm{c}^{\prime}, \mathrm{f}^{\prime}\right)$ large droplets.

10 FIG. 3. Temporal texture change of $\mathrm{N}_{\mathrm{TB}}$ droplets undergoing photo-induced transition, on ( $\mathrm{a}$, 11 a') bare glass, (b, b') rubbed PVA, and (c, c') homeotropic PI substrates. Temperature was 12 stationary at $\sim 60^{\circ} \mathrm{C}$. (a, b, c) The changes with time after switching on UV, and (a', b', c') 13 after switching off UV. Time intervals are given between the images. See also the 14 corresponding movies available in SM1.

FIG. 4. Structural analysis for $\mathrm{N}$ droplet on bare glass. (a) POM observed with a tint plate.

17 The directions of polarisers and the optic axis of the tint plate are shown together. The blue and yellow ellipses in the leftmost figure represent molecular orientations deduced from the

19 colour change at the edges in these directions. (a') Simulation result using the Jones matrix

20 method. The calculation was done with the director field described by Eq. (2). (b-b')

21 Schematic representation of the director field deduced from the experimental and simulation

22 results. The ellipses show the point-by-point distributions of the $\mathrm{N}$ directors projected onto

23 the plane. The colour gradation of these ellipses from red to blue represents the director tilt 
1 from up to down against the plane. (c-c') Computer-generated 3D cartoons based on Eq. (2).

3 FIG. 5. Structural analysis for $\mathrm{N}$ droplet on PVA-coated glass. (a) POM observed with a tint

4 plate. The directions of polarisers and the optic axis of the tint plate are shown together. The

5 green arrows show the rubbing direction. The blue and yellow ellipses in the leftmost figure

6 represent molecular orientations deduced from the colour change at the edges in these

7 directions. (a') Simulation result using the Jones matrix method. The calculation was done

8 with the director field described by Eq. (3). (b-b') Schematic representation of the director

9 field deduced from the experimental and simulation results. The ellipses show the point-by-

10 point distributions of the $\mathrm{N}$ directors projected onto the plane. The colour gradation of these

11 ellipses from red to blue represents the director tilt from up to down against the plane. $\left(\mathrm{c}-\mathrm{c}^{\prime}\right)$

12 Computer-generated 3D cartoons based on Eq. (3).

13

14 FIG. 6. Structural analysis for N droplet on homeotropic PI-coated glass. (a) POM observed

15 with a tint plate. The directions of polarisers and the optic axis of the tint plate are shown

16 together. (a') Simulation result using the Jones matrix method. The calculation was done

17 with the director field described by Eq. (4). (b-b') Schematic representation of the director

18 field deduced from the experimental and simulation results. The ellipses show the point-by-

19 point distributions of the $\mathrm{N}$ directors projected onto the plane. The colour gradation of these

20 ellipses from red to blue represents the director tilt from up to down against the plane. (c-c')

21 Computer-generated 3D cartoons based on Eq. (4).

22

23 FIG. 7. (a) A model for the stripe domains of the spherical-cap $\mathrm{N}_{\text {тв }}$ droplets on the bare glass 
1 and the rubbed PVA substrates. In this model, pseudo-layer nature of nano-pitched helix is

2 the cause of the stripes and undulations. This model is borrowed from Ref. 11 [11 Lopez-

3 Leon], made for the similar structure with layer undulations in thick smectic shells. Dotted

4 lines show the direction of the pseudo-layers. See the caption of Fig. 4-6 for the explanation

5 of the ellipses. (b) The pitch of stripes $d$ and the droplet height $h$ plotted against the droplet

6 radius $R$. The measurement was performed at $\sim 60^{\circ} \mathrm{C}$. In all the cases, the data are well fitted

7 by linear functions, and interestingly $d$ coincides with $h$. The slope values $d / R$ and $h / R$ are

8 summarized in Table 1. (c) The $R$ dependence of the radius $\left(R_{\mathrm{c}}\right)$ of the inner circular domain

9 appearing in the large spherical-cap $\mathrm{N}_{\mathrm{TB}}$ droplet on the homeotropic PI. The measurement

10 was performed at $\sim 60^{\circ} \mathrm{C}$. The corresponding droplet height $h$ is also plotted. In the whole $R$

11 range, $R_{\mathrm{c}}$ is smaller than $h$, and it shows a non-linear dependence with the droplet radius. 
(a) Top

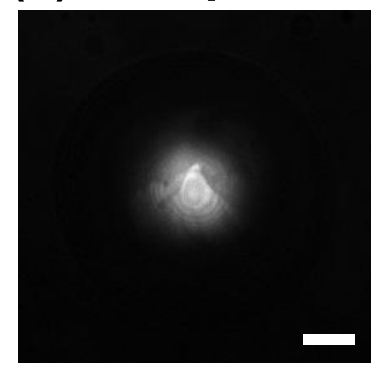

(b) Mid-Plane

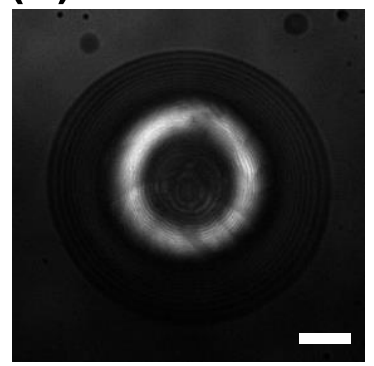

(c) Bottom

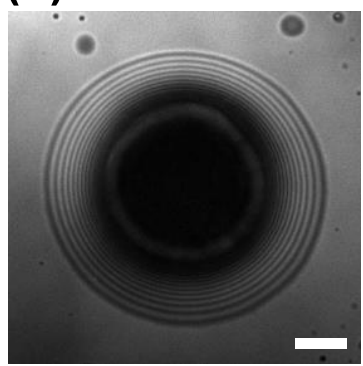

Horizontal Cross-Sections

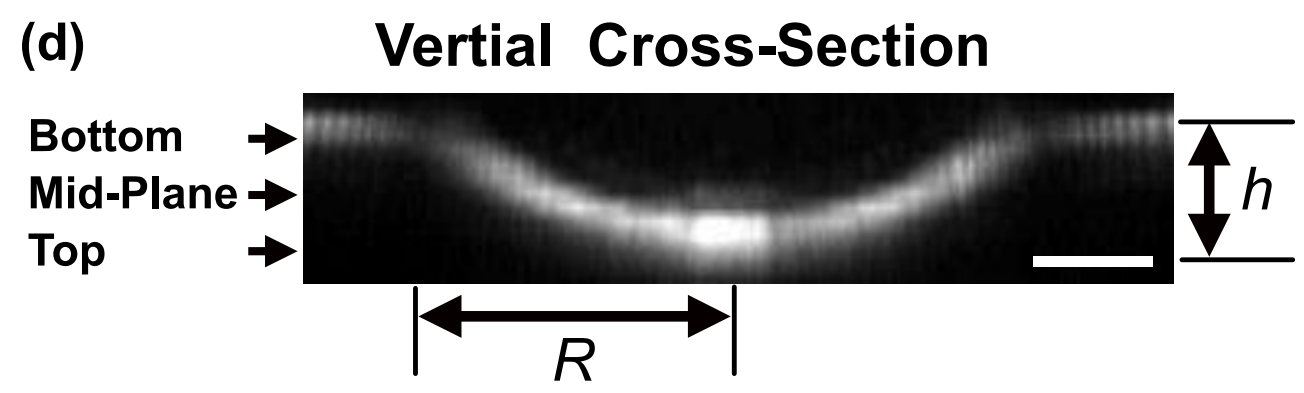

(e)

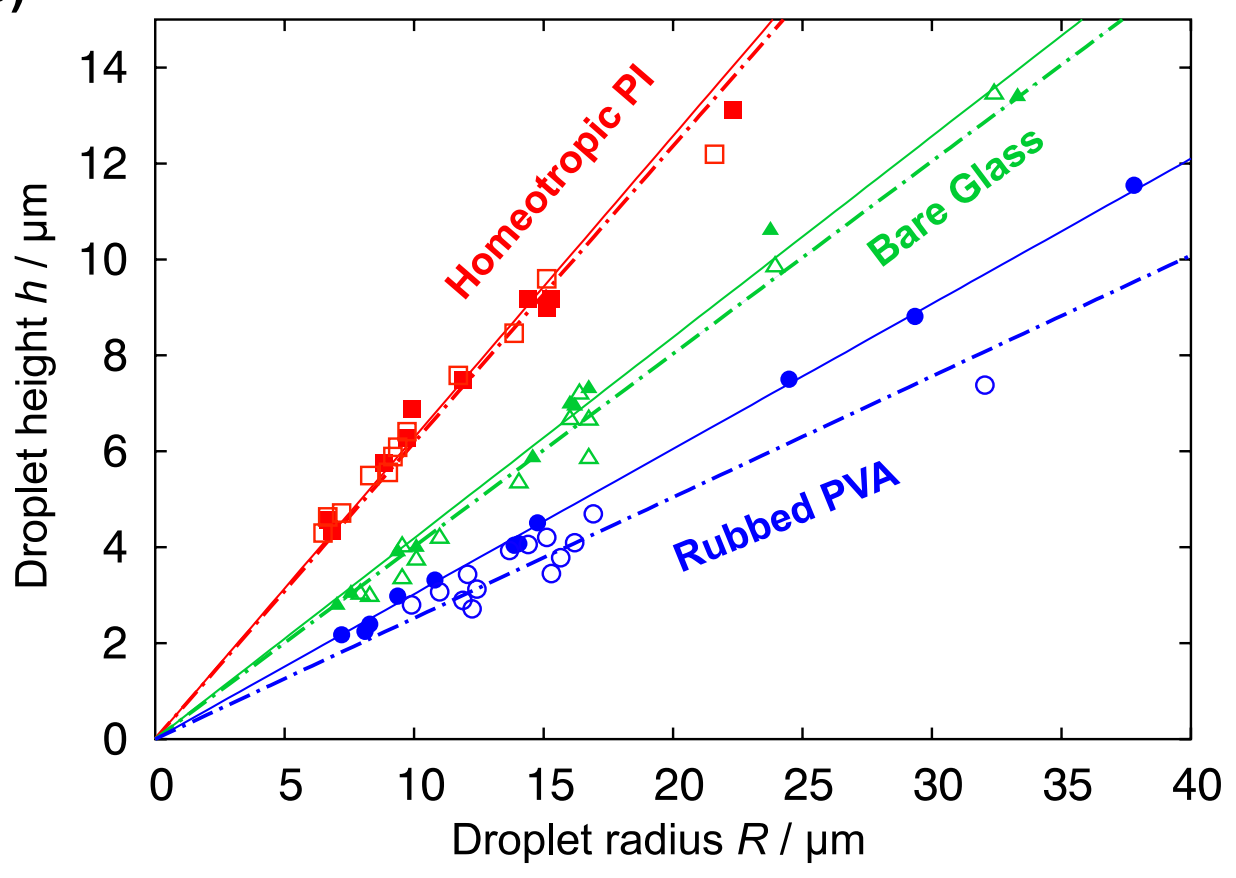

Figure 1 


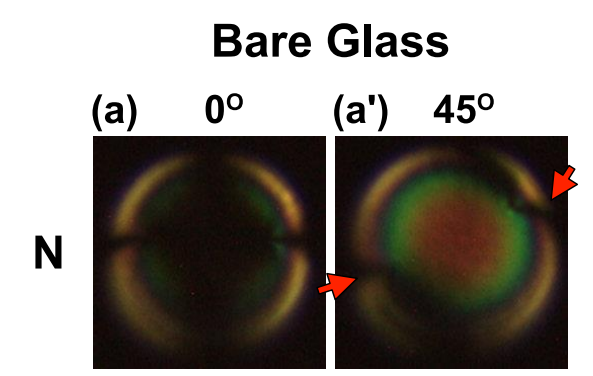

Rubbed PVA

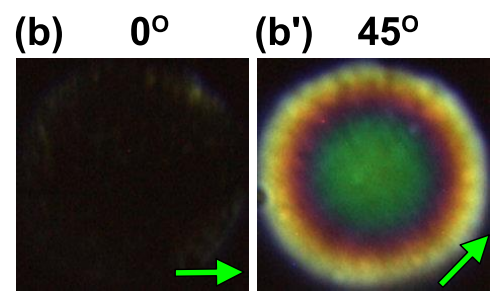

(d) $0^{\circ}$

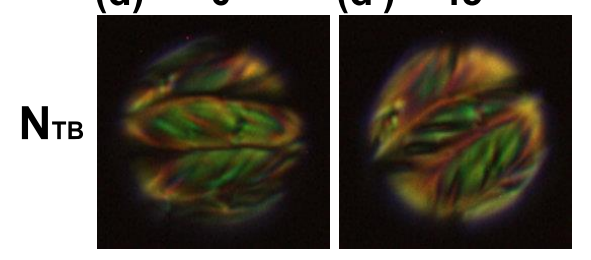

(e) $0^{\circ}$

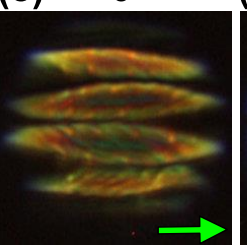

(e') $45^{\circ}$

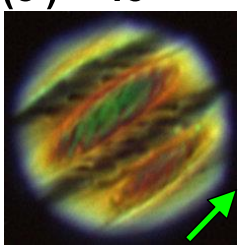

(c) Smaller

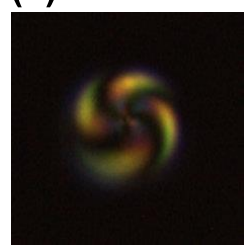

(f) Smaller

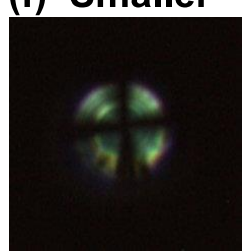

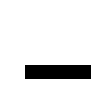

$10 \mu \mathrm{m}$

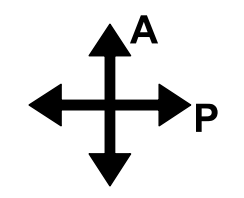

Figure 2

4 
Bare Glass

(a)
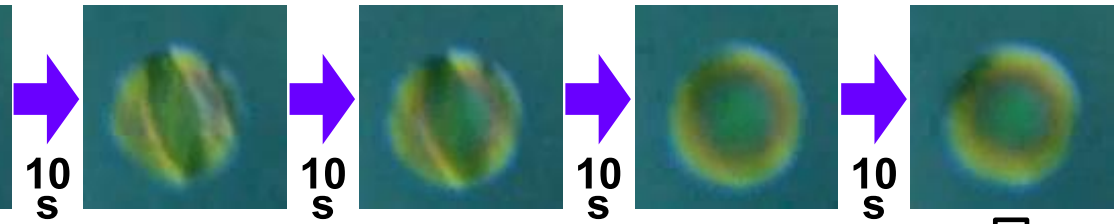

(a')
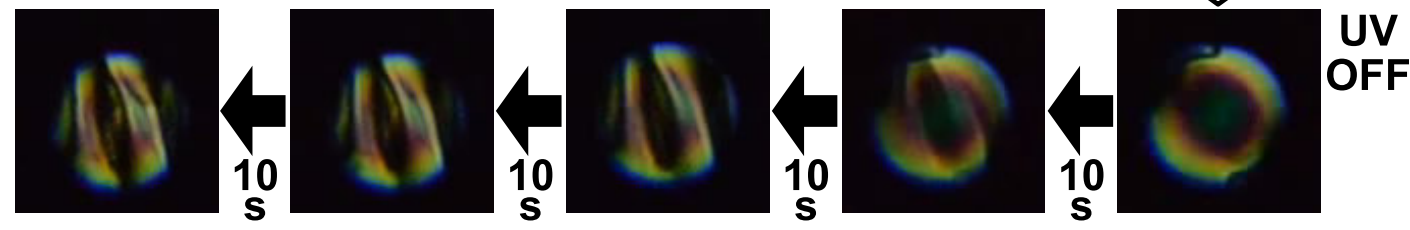

Rubbed PVA

(b)
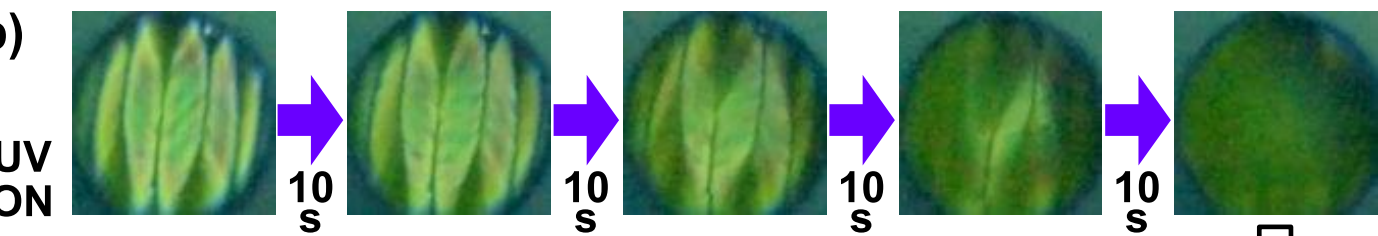

(b')
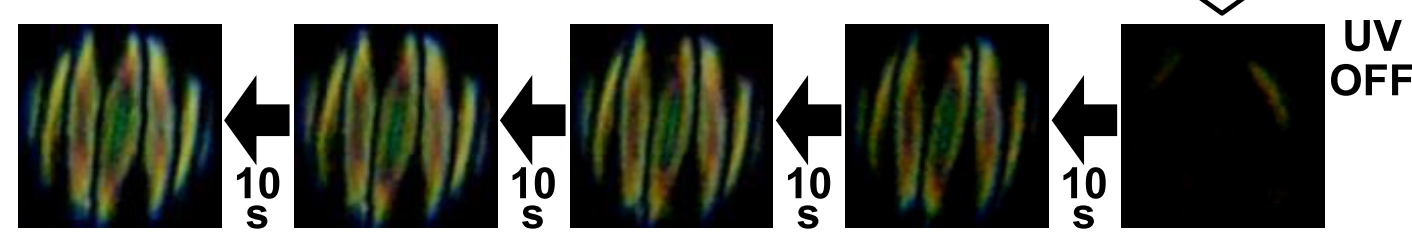

Homeotropic PI

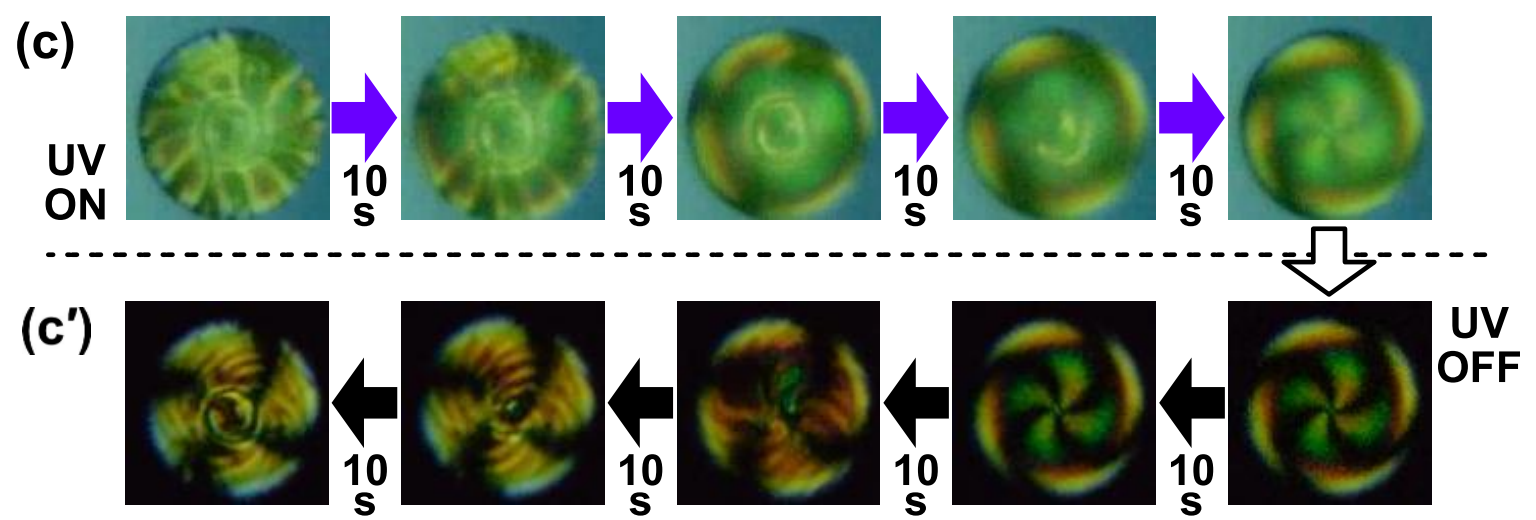

Figure 3 
(a)
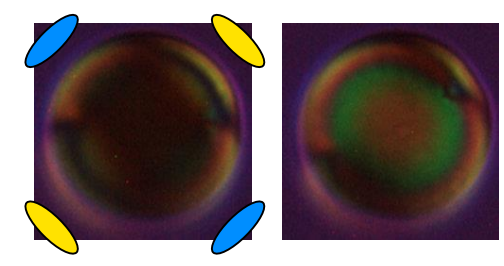

(a')
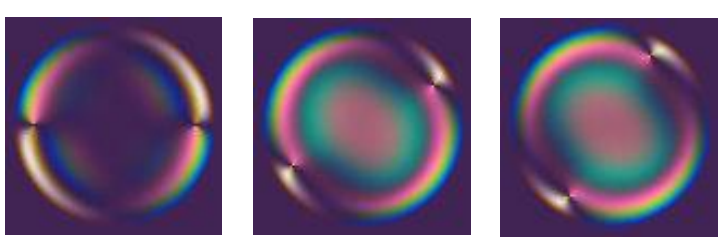

(b)

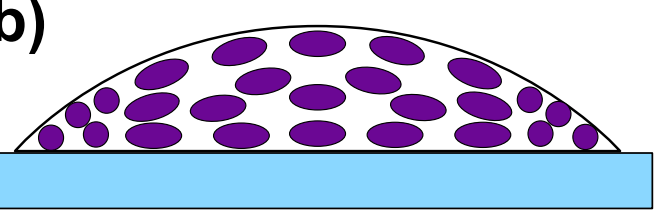

(b')

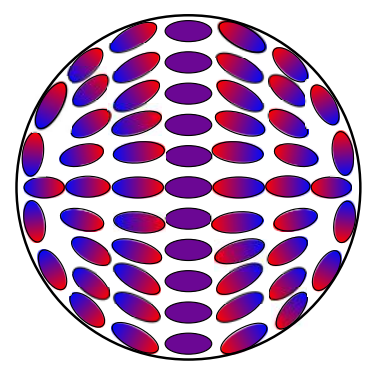

(b")

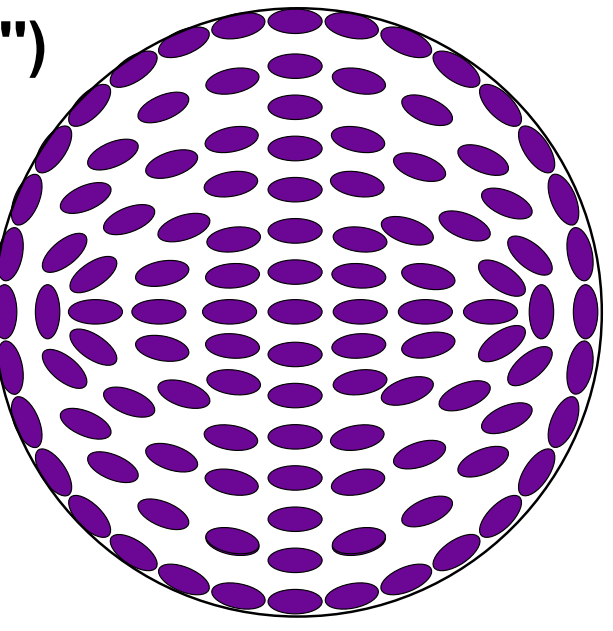

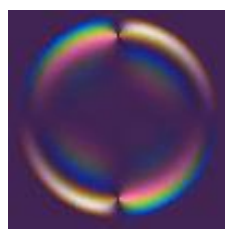
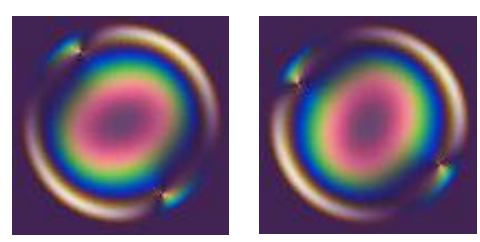

(c)
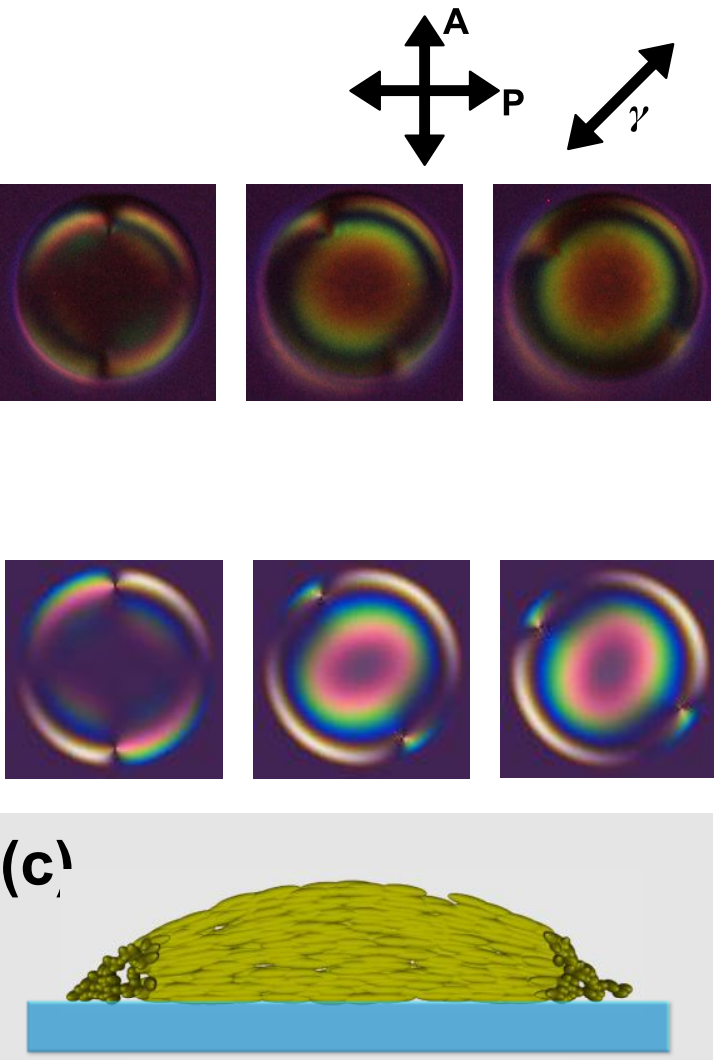

(c')

(c")
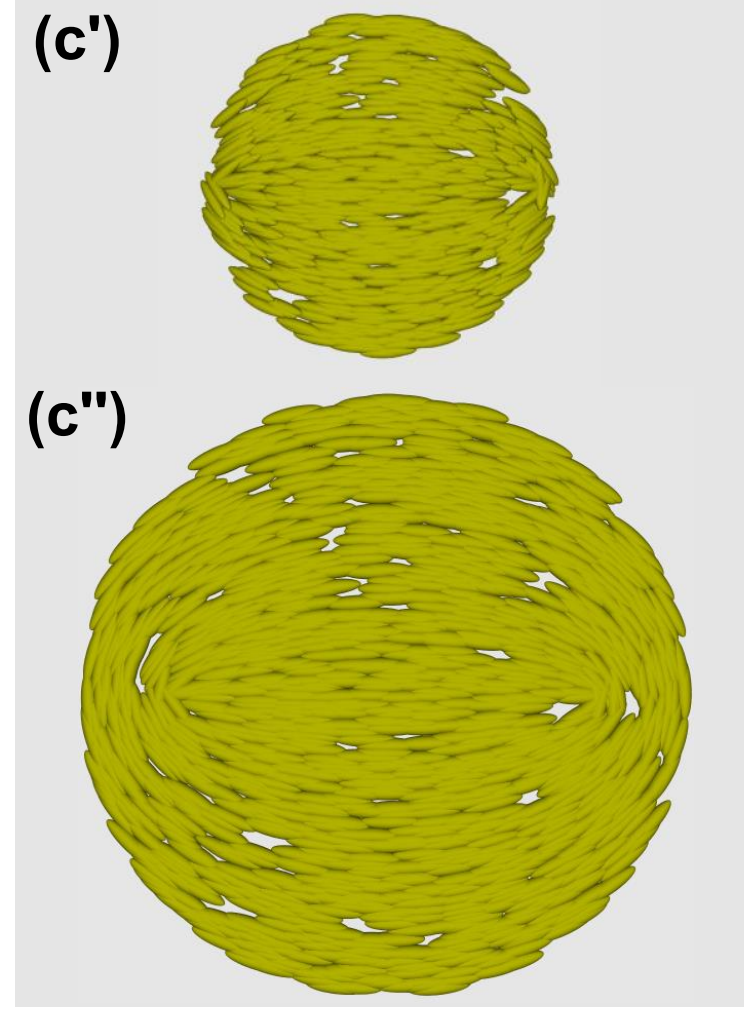

Figure 4 
(a)
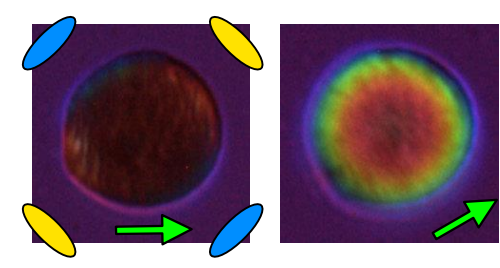

(a')
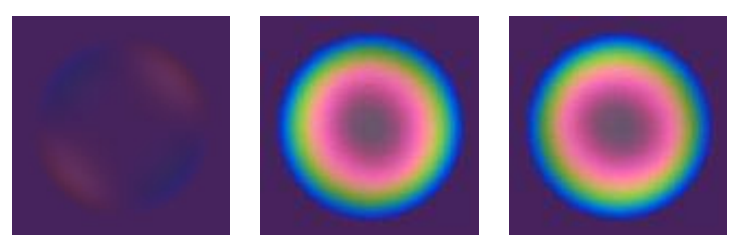

(b)

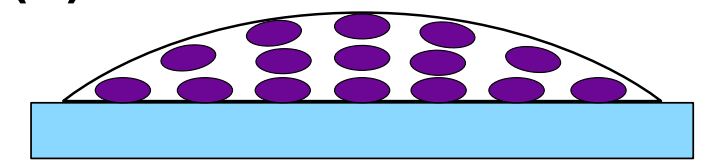

(b')

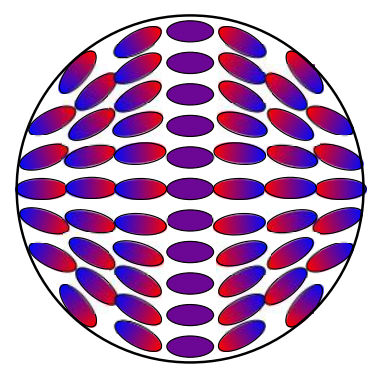

(b")

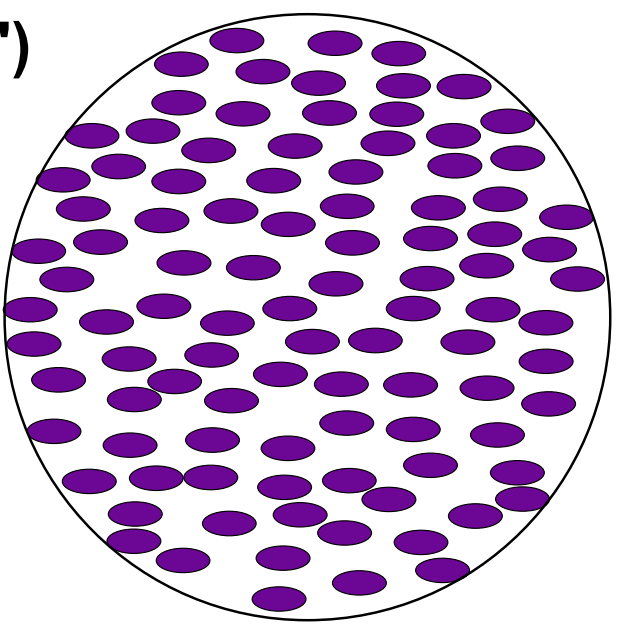

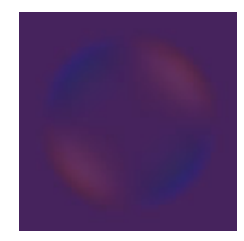
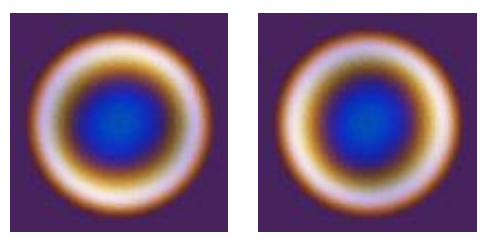

(c)
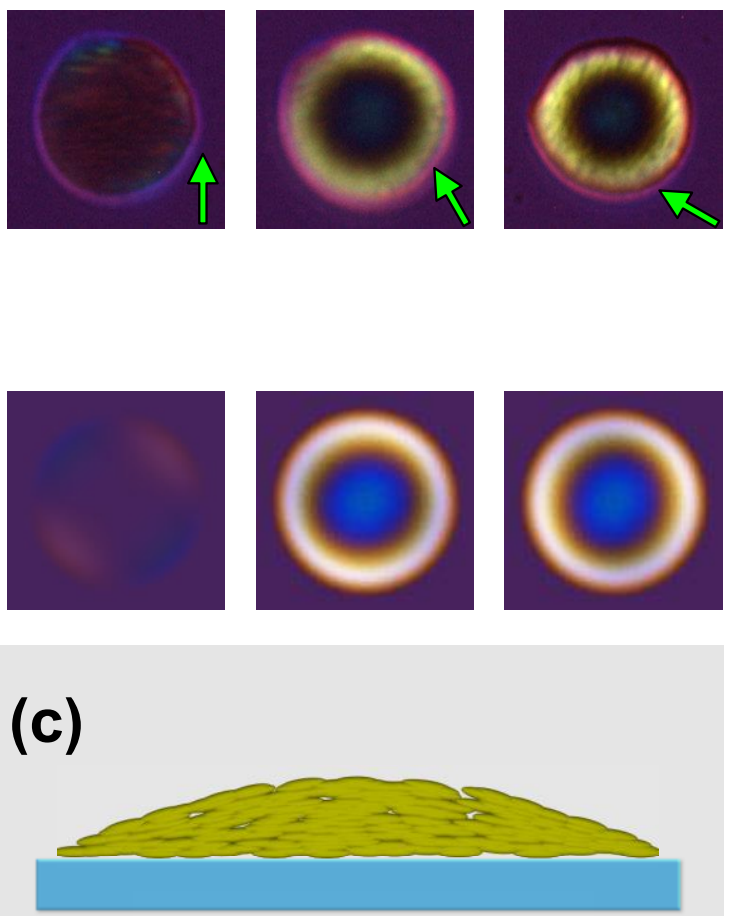

(c')
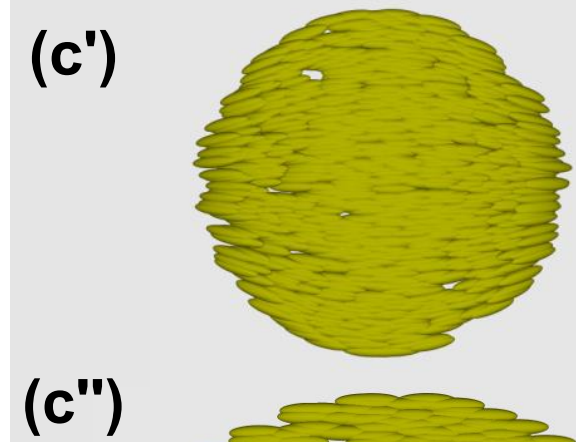

(c")

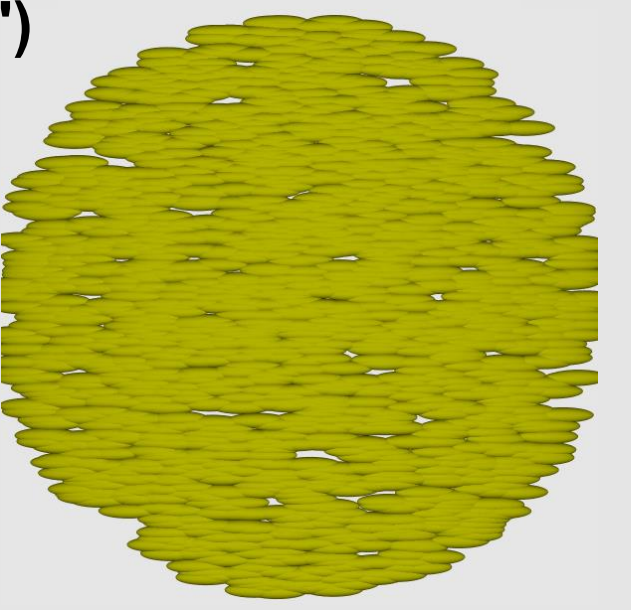

Figure 5 
(a)

(a')
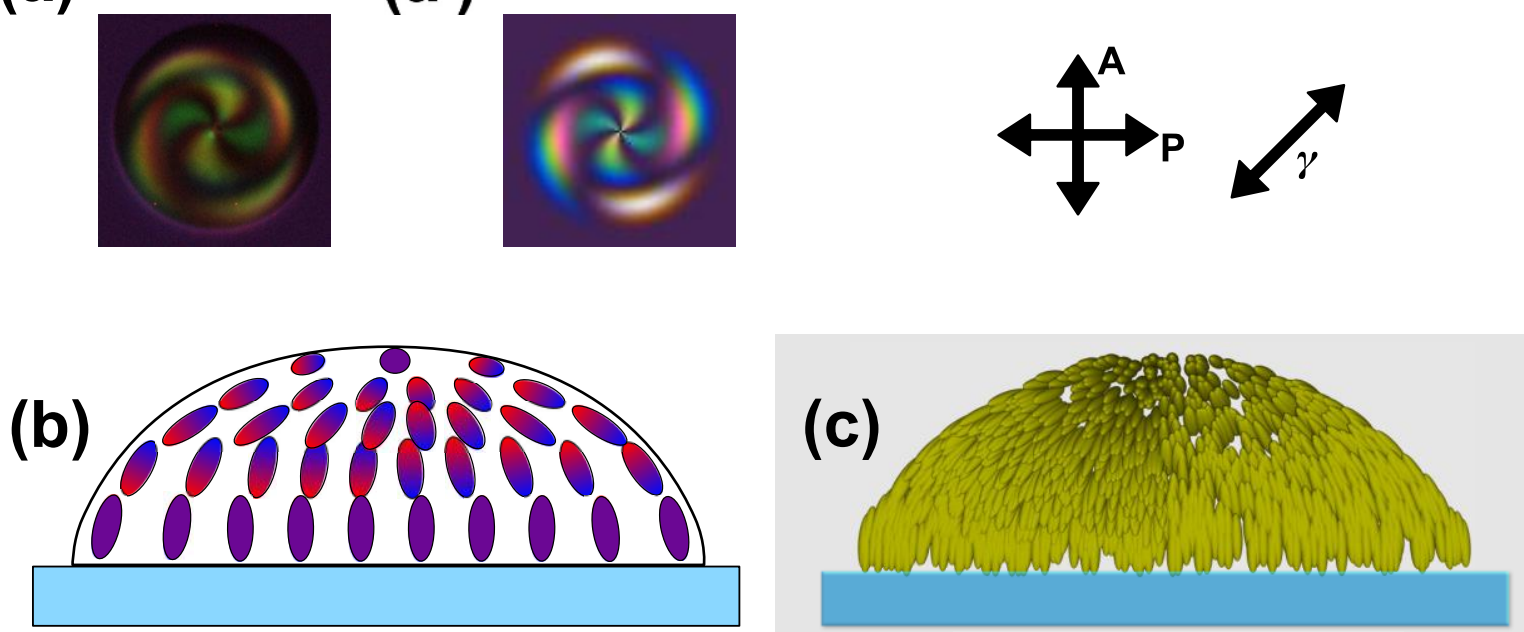

(b')

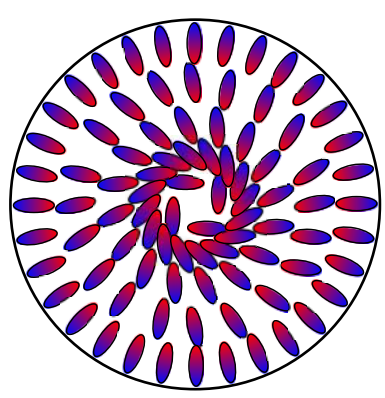

(b")
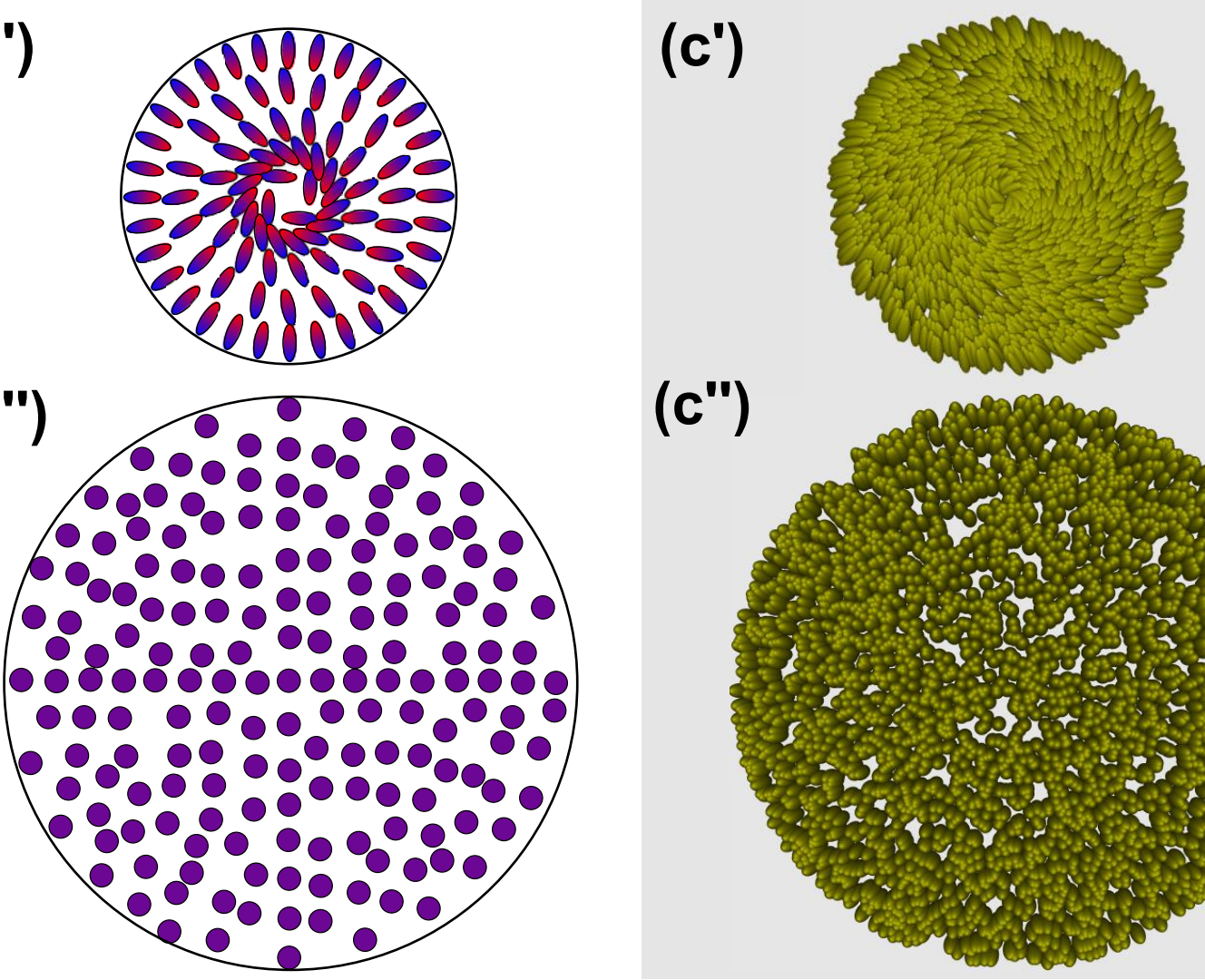

(c")

Figure 6 
(a)
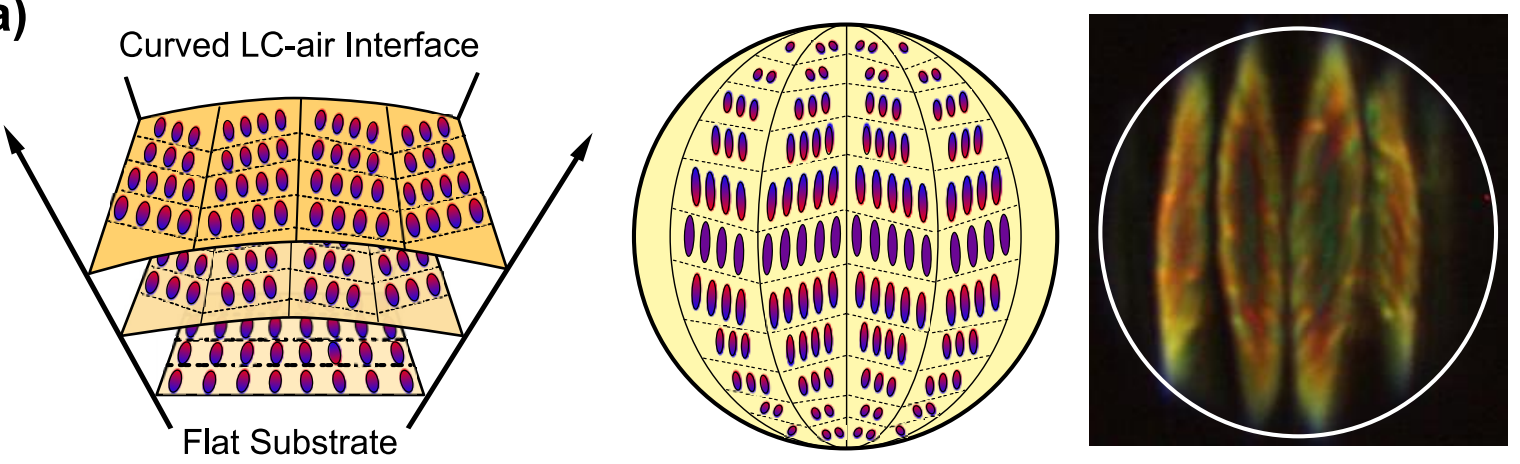

(b)

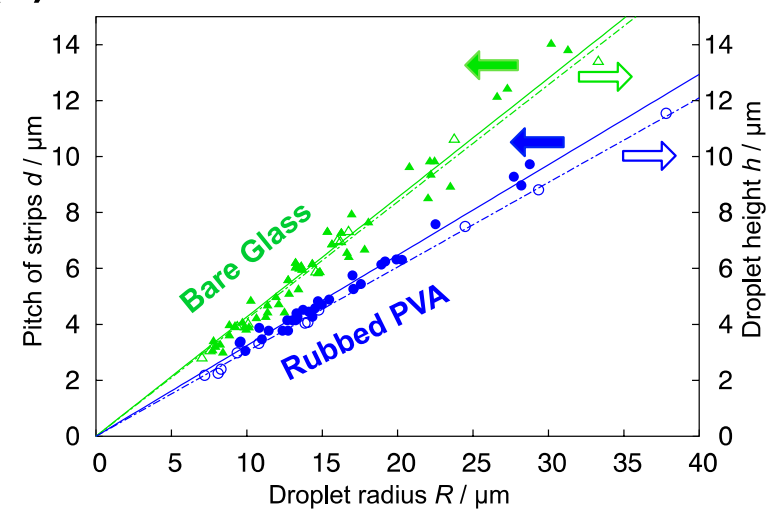

(c)

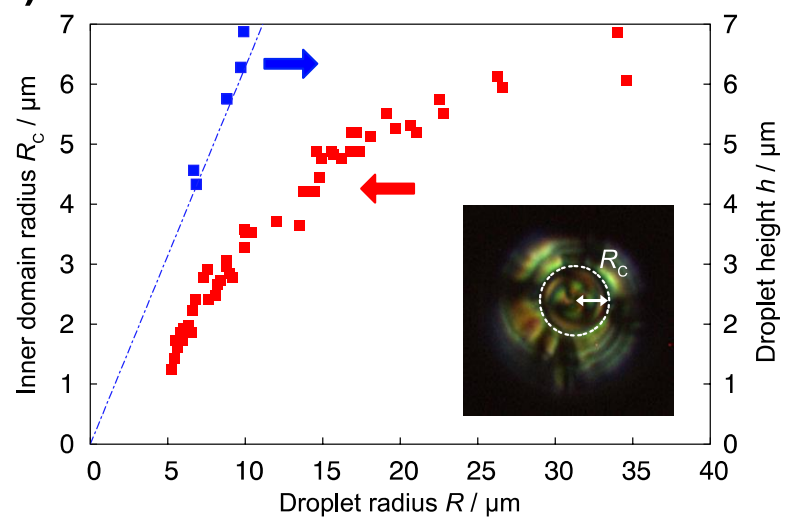


Table I

\begin{tabular}{lccc}
\hline & $h / R$ & $\theta_{c}[\mathrm{rad}]$ & $d / R$ \\
\hline \hline Bare glass (N) & 0.40 & 0.76 & \\
Bare glass (NTB) & 0.42 & 0.79 & 0.43 \\
PVA (N) & 0.25 & 0.49 & \\
PVA (NTB) & 0.30 & 0.58 & 0.32 \\
JALS204 (N) & 0.62 & 1.11 & \\
JALS204 (NTB) & 0.63 & 1.12 & \\
\hline
\end{tabular}

